\title{
Study on Polysaccharide by the Fluorescence Method. II. Micro-Brownian Motion and Conformational Change of Amylose in Aqueous Solution
}

\author{
Shinichi Kitamura, Harunobu YunoKawa, Sanae Mitsuie, \\ and Takashi KUGE \\ Laboratory of Biopolymers, Department of Agricultural Chemistry, \\ Kyoto Prefectural University, Sakyo-ku, Kyoto 606, Japan.
}

(Received March 23, 1981)

\begin{abstract}
The segmental motion of amylose in aqueous solution and the conformational transition with temperature and $\mathrm{pH}$ were studied by the fluorescence polarization method. We prepared two types of fluorescent conjugates of amylose: amylose conjugated with fluorescein randomly throughout chain (F-Amylose) and amylose conjugated locally on a terminal segment ( $t$ F-Amylose). The value of the rotational relaxation time for $t$-F-Amylose was much smaller than that for F-Amylose. This result suggests that a terminal segment undergoes a more rapid microBrownian motion than interior segments. Polarization curves of these conjugates gradually departed from the Perrin equation when the temperature was raised. F-Amylose showed a gradual departure above $45^{\circ} \mathrm{C}$ but $t$-F-Amylose showed a departure at room temperature. This indicates that the conformational transition occurs first in terminal segments and then extends to the interior segments with increasing temperature. The effect of $\mathrm{pH}$ on the polarization was also examined. When measured as a function of $\mathrm{pH}$, fluorescence depolarization increased pronouncedly in the alkaline region. In contrast to changing temperature, depolarization began to increase at the same $\mathrm{pH}$ for the two amylose conjugates.
\end{abstract}

KEY WORDS Amylose / Fluorescent Conjugate / Fluorescence Depolarization / Segmental Motion / Terminal Segment / Interior Segment / Deformed Helix / Conformational Transition /

The conformation of amylose in an aqueous solution has been intensively studied by means of hydrodynamic methods. ${ }^{1-4}$ These studies suggest that amylose behaves as a random coil in an aqueous solution. It is therefore considered that an amylose chain takes on many different shapes and fluctuates continuously among these. However, we note that hydrodynamic measurements can provide information only about the overall character of the molecule. The local conformation of amylose in an aqueous solution is a matter of dispute in spite of extensive investigations. ${ }^{5,6}$

The fluorescence polarization method is expected to provide information on the segmental motion and the conformation of a polymer chain to which a fluorescent residue is attached. Thus, when a polymer chain undergoes a conformational transition, the course of the transition may be followed by measuring the fluorescence polarization of the attached dye residue. ${ }^{7-9}$

The purpose of the present study is twofold: to obtain information on segmental motion and the conformation of amylose about the attached dye residue and to study the conformational transition of amylose induced by temperature and $\mathrm{pH}$ changes, both by the fluorescence polarization method. We prepared two types of fluorescent conjugates of amylose: amylose conjugated randomly with fluorescein residues throughout the chain and amylose conjugated only at a terminal segment. These fluorescent conjugates enabled us to investigate the differences in segmental mobility and conformation between the interior and terminal segments of amylose. 


\section{S. Kitamura et al.}

\section{EXPERIMENTAL}

\section{Basic Principle of Synthetic Amylose Preparation}

Phosphorylase catatyzes the reaction represented by,

$$
\begin{gathered}
n \cdot(\text { glucose } 1-\text { phosphate })+\text { primer } \rightleftarrows \\
\text { amylose }+n \cdot(\text { orthophosphate })
\end{gathered}
$$

When glucose 1-phosphate is in excess, the forward reaction occurs predominantly. It is well known that this synthetic reaction takes place in a mode all the primer chains grow at an approximately equal rate. ${ }^{10}$ Hence, one product of this reaction may be nearly monodisperse synthetic amylose. The molecular weight of the product can be calculated from the amount of phosphate liberated in the reaction mixture and the concentration of the primer used.

\section{Preparation of F-Amylose}

Maltopentaose was obtained by fractionation of acid hydrolyzates of $\beta$-cyclodextrin by cellulose column chromatography. The potato phosphorylase used was a gift from Professor Toshio Fukui of Osaka University. The enzyme was free from other carbohydrase activity. ${ }^{11}$ The reaction mixture $\left(4 \times 10^{-5} \mathrm{M}\right.$ maltopentaose, $0.1 \mathrm{M}$ glucose 1-phosphate, 120 units of phosphorylase and $0.01 \mathrm{M}$ maleate buffer in total volume of $200 \mathrm{ml}$ ) was incubated at $35^{\circ} \mathrm{C}$ for $450 \mathrm{~min}$. The reaction was stopped by the addition of $100 \mathrm{ml}$ of $5 \%$ trichloroacetic acid, followed by neutralization with $\mathrm{KOH}$. This reaction mixture was dialyzed against dimethylsulfoxide (DMSO)-water $(1: 4, \mathrm{v} / \mathrm{v})$ solution at $35^{\circ} \mathrm{C}$ for 2 days. After dialysis, $250 \mathrm{ml}$ of ethanol was added to precipitate the amylose produced. The precipitate was washed five times with $500 \mathrm{ml}$ of ethanol, twice with $300 \mathrm{ml}$ of ether and dried overnight in vacuo at $70^{\circ} \mathrm{C}$. The amylose prepared by this procedure had a number-average molecular weight of 110,000 . The amylose was shown to be nearly monodisperse by gel permeation chromatography using a Sepharose CL-4B (Pharmacia, Uppsala).

Conjugation with fluorescein was carried out according to the method of Belder described previously ${ }^{7,12)}$ The degree of substitution with dye was regulated by changing the amount of dye added to the reaction mixture. The conjugate obtained, FAmylose, is considered to have fluorescein residues randomly on the chain.

\section{Preparation of $t$-F-Amylose}

Using the maltopentaose-fluorescein conjugate as a primer in the synthetic reaction catalyzed by phosphorylase, we obtained amylose specifically modified with a fluorescein residue near the chain end. The name of this type of conjugate is $t-\mathrm{F}$ Amylose. A reaction mixture $\left(2.6 \times 10^{-5} \mathrm{M}\right.$ fluorescein-maltopentaose conjugate, $0.1 \mathrm{M}$ glucose 1-phosphate, 30 units of phosphorylase and $0.01 \mathrm{M}$ maleate buffer in total volume of $50 \mathrm{ml}$ ) was incubated at $35^{\circ} \mathrm{C}$ for $300 \mathrm{~min}$. The enzymatic reaction was stopped by dialysis. Thereafter, the reaction mixture was dealt with in the same way as F-Amylose.

\section{Elution pattern of $t-F$-Amylose by Sepharose $C L-4 B$}

Gel permeation chromatography of $t$-F-Amylose was performed using a Sepharose CL-4B in order to investigate molecular weight distribution. The chromatographic column used was $70 \mathrm{~cm}$ long and $2.1 \mathrm{~cm}$ in diameter. The elution solvent was $0.05 \mathrm{M}$ aqueous $\mathrm{KOH}$ solution. The flow rate was regulated to $0.33 \mathrm{ml} \mathrm{min}^{-1}$. The parameters $V_{0}$ and $V_{\mathrm{t}}$ were determined using blue dextran (Pharmacia, Uppsala) and $\mathrm{KCl}$, respectively. $8 \mathrm{mg}$ of $t$-FAmylose were dissolved in $0.5 \mathrm{ml} \mathrm{DMSO}$, diluted to $5 \mathrm{ml}$ with distilled water, and $2 \mathrm{ml}$ of this solution were charged on the column. Each $5 \mathrm{ml}$ of eluent was collected. The carbohydrate content in each fraction was determined by the phenol-sulfuric acid colorimetric method ${ }^{13}$ and fluorescent intensity at $520 \mathrm{~nm}$ was measured using a Shimadzu spectrofluorometer RF-502. For calibration of the column, a series of synthetic amylose with molecular weights of $0.88,2.6,5.8,11,13,36$, and $99 \times 10^{4}$ were used.

The elution pattern of $t$-F-Amylose is shown in Figure 1. Two peaks are seen at $V_{0}$ and $150 \mathrm{ml}$ on the total carbohydrate curve, but only one peak at $150 \mathrm{ml}$ in the fluorescence intensity curve. The carbohydrate which collected about $V_{0}$ exhibited virtually no fluorescence and may have been synthesized from maltopentaose without fluorescein. The molecular weight of the amylose conjugated with fluorescein was estimated to be 130,000 from the elution volume for the maximum fluorescence intensity.

\section{Methods}

Fluorescence polarization and fluorescence life- 
Polysaccharide Study by the Fluorescence Method: II.

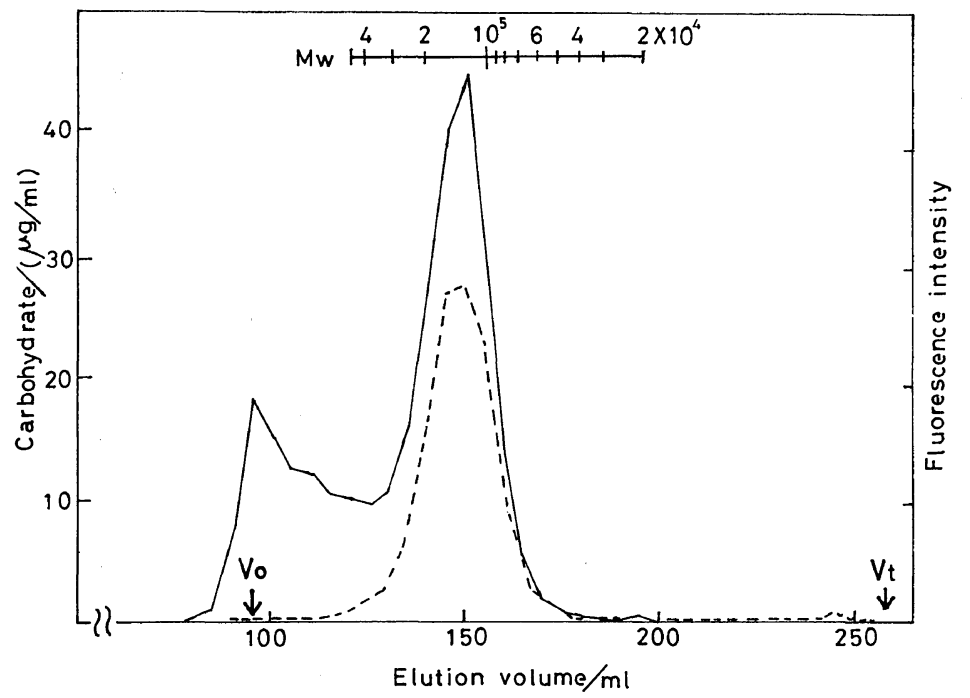

Figure 1. Elution pattern of $t$-F-Amylose by GPC using Sepharose CL-4B. (-), carbohydrate; (---), fluorescence intensity.

time measurements were performed as described before. $^{7}$

The mean rotational relaxation time, $\langle\rho\rangle$, of the kinetic unit carrying the emission oscillator was computed from an equation of the Perrin type, ${ }^{7,14}$

$$
\frac{1}{P}-\frac{1}{3} \approx\left(\frac{1}{P_{0}^{\prime}}-\frac{1}{3}\right)\left(1+\frac{3 \tau}{\langle\rho\rangle}\right)
$$

where $P_{0}{ }^{\prime}, \tau$ and $\langle\rho\rangle$ have the same meaning as in the previous paper. ${ }^{7}$

Since F-Amylose and $t$-F-Amylose were insoluble in water, they were dissolved in a small amount of DMSO and diluted with a phosphate buffer solution $(0.01 \mathrm{M}, \mathrm{pH} 7)$. The polymer concentrations of the final solutions were from $0.015-0.08 \%$. In the $\mathrm{pH}$ profile experiments, dilution was performed with distilled water and the $\mathrm{pH}$ of the solution was adjusted by adding an aqueous $\mathrm{KOH}$ solution of appropriate concentration. The viscosities of water and sucrose solution were taken from International Critical Tables ${ }^{15}$ and Kagaku Binran. ${ }^{16}$

\section{RESULTS}

Some Properties of F-Amylose and t-F-Amylose

The absorption spectra of F-Amylose and $t$-FAmylose were similar to that of fluorescein except for a red shift from 2 to $4 \mathrm{~nm}$. The emission spectra of these conjugates were also similar to that of fluorescein except for a red sift of about $5 \mathrm{~nm}$. The degree of substitution of dye was estimated spectrophotometrically using methyl $\mathrm{N}$-fluoresceinlythiocarbamate as a reference. The degree of substitution for $t$-F-Amylose in Table I was estimated, excluding amylose collected about $V_{0}$ in Figure 1 . The degrees of substitution for F-Amylose and $t$-F-Amylose were essentially the same. These conjugates have nearly the same fluorescence lifetimes as that of the free dye. The results are summarized in Table I.

Comparison of $\langle\rho\rangle$ values for $F$-Amylose and $t-F$ Amylose

Figure 2 shows plots of $1 / P$ versus $T / \eta$ for $\mathrm{F}$ Amylose and $t$-F-Amylose at $25^{\circ} \mathrm{C}$. The solvent viscosity was varied by the addition of sucrose. Since $P_{0}{ }^{\prime}$ can be estimated from the ordinate intercept obtained by linear extrapolation, the value of $\langle\rho\rangle / \tau$ for each conjugate can be calculated by eq 1 . Combining this value with the fluorescence lifetime, we can calculate $\langle\rho\rangle$. Table II shows the values of $1 / P_{0}{ }^{\prime},\langle\rho\rangle / \tau, \tau$, and $\langle\rho\rangle$ for F-Amylose and $t$-F-Amylose. The $\langle\rho\rangle$ value for F-Amylose is nearly twice as large as that for $t$-F-Amylose. Therefore, it may be said that the terminal segments 


\section{S. Kitamura et al.}

Table I. Some properties of F-Amylose and $t$-F-Amylose ${ }^{\mathrm{a}}$

\begin{tabular}{lccccc}
\hline & Molecular & & \multicolumn{2}{c}{ Wavelength/nm } & Fluorescence \\
weight & DS & & $\begin{array}{c}\text { Absorption } \\
\text { maximum }\end{array}$ & $\begin{array}{c}\text { Emission } \\
\text { maximum }\end{array}$ & \\
\hline Fluorescein & 376 & - & 491 & 513 & 4.3 \\
F-Amylose & 110000 & 0.001 & 495 & 518 & 4.4 \\
$t$-F-Amylose & 130000 & 0.0009 & 493 & 519 & 4.5 \\
\hline
\end{tabular}

a DS, degree of substitution with dye is defined as the fraction of glucose residues substituted. Absorption and

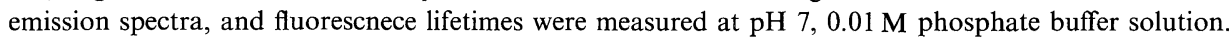

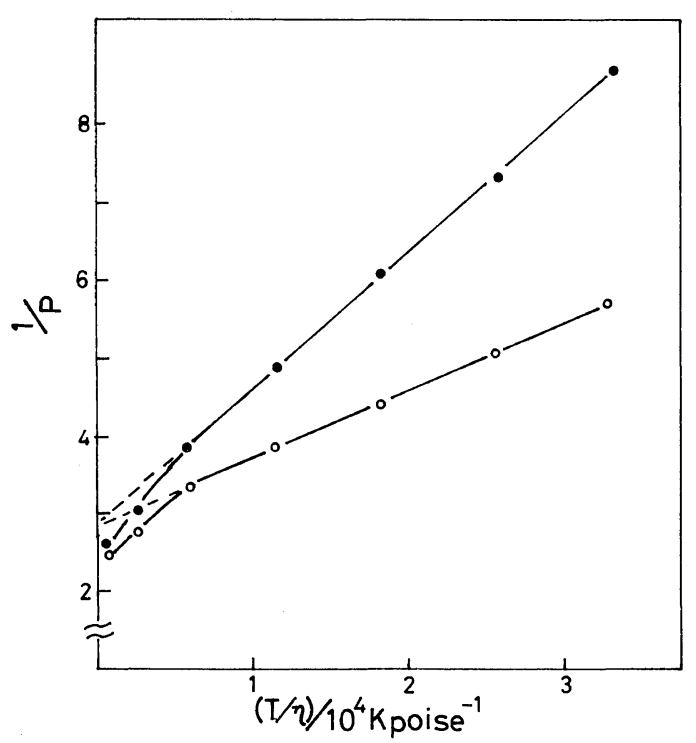

Figure 2. Plots of $1 / P v s . T / \eta$ for F-Amylose $(O)$ and $t$-F-Amylose (O) in $0.01 \mathrm{M}$ phosphate buffer solution of $\mathrm{pH} 7$ at $25^{\circ} \mathrm{C}$. The solvent viscosity was varied by the addition of sucrose.

show more rapid segmental motion than the interior segments.

\section{Effect of Temperature on the Polarization}

Figure 3 shows the effect of temperature on the polarization of F-Amylose and $t$-F-Amylose. It is seen that significant upward curvatures are present in these Perrin plots. This behavior on the part of FAmylose is attributed to the accelerated loosening of the internal structure of the amylose chain with an increase in temperature. ${ }^{7}$ Although for $\mathrm{F}$ Amylose the upward curvature is seen at temperatures above about $45^{\circ} \mathrm{C}, t$-F-Amylose begins to show the same behaivor at as low a temperature as $25^{\circ} \mathrm{C}$.
Table II. Comparison of $1 / P_{0}{ }^{\prime},\langle\rho\rangle / \tau, \tau$, and $\langle\rho\rangle$ values for F-Amylose and $t$-F-Amylose in a $0.01 \mathrm{M}$ phosphate buffer solution at $25^{\circ} \mathrm{C}$

\begin{tabular}{lcccc}
\hline \multicolumn{1}{c}{ Conjugate } & $1 / P_{0}{ }^{\prime}$ & $\langle\rho\rangle / \tau$ & $\tau / \mathrm{ns}$ & $\langle\rho\rangle / \mathrm{ns}$ \\
\hline F-Amylose & 2.79 & 2.54 & 4.4 & 11 \\
$t$-F-Amylose & 2.90 & 1.35 & 4.5 & 6.1 \\
\hline
\end{tabular}

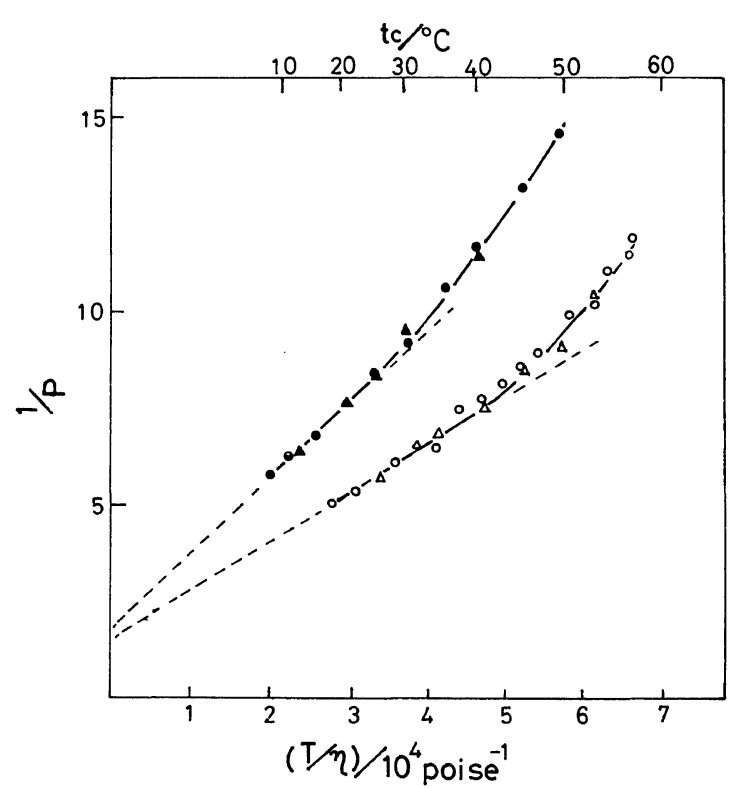

Figure 3. Effect of temperature on the polarization of F-Amylose $(O, \triangle)$ and $t$-F-Amylose $(\boldsymbol{O}, \boldsymbol{\Delta})$. The temperature increased $(O, \mathbf{O})$ and subsequently decreased $(\triangle, \boldsymbol{\Delta})$.

This difference suggests that the conformational transition of amylose first occurs in the terminal segments and then extends to all segments with increasing temperature. 
Polysaccharide Study by the Fluorescence Method. II.

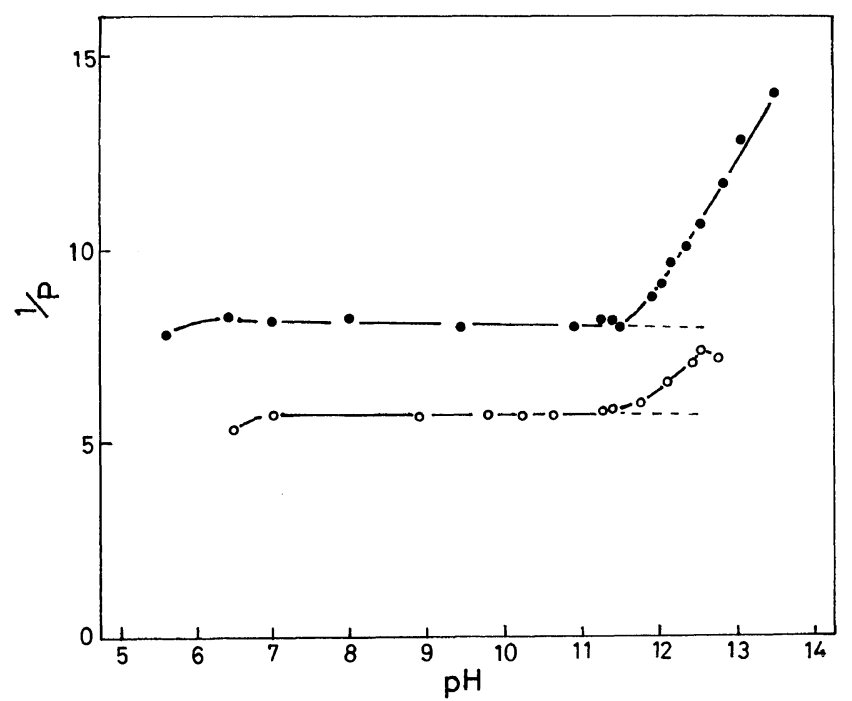

Figure 4. Effect of $\mathrm{pH}$ on the polarization of fluorescence for $\mathrm{F}$-Amylose $(\mathrm{O})$ and $t$-F-Amylose $(\bigcirc)$ at $25^{\circ} \mathrm{C}$.

\section{Effect of $\mathrm{pH}$ on the Polarization}

Figure 4 shows the $\mathrm{pH}$ dependence of fluorescence polarization of F-Amylose and $t$-F-Amylose. The 1/P values for these conjugates stay constant at a $\mathrm{pH}$ below 11.5, but above this, they increase with increasing $\mathrm{pH}$. The fluorescence lifetimes of the conjugates in the alkaline region above $\mathrm{pH} 12$ were found experimentally to be essentially the same as those in a neutral solution.

It has been shown that amylose in an aqueous solution exhibits considerable change in intrinsic viscosity and optical rotation in the region of $\mathrm{pH}$ between 11 and 13, and this behavior has been interpreted in terms of the conformational change caused by increasing negative charges on the polymer chain. ${ }^{17-19}$ It can be concluded that the increase in the depolarization observed here is caused by the same conformational transition. In contrast to the temperature dependence, the transition of either conjugate becomes appreciable at nearly the same $\mathrm{pH}$.

\section{DISCUSSION}

It was found in this study that the terminal segment of an amylose chain undergoes more rapid micro-Brownian motion than the interior segment. Uchida et al. reported a similar finding for polystyrene. ${ }^{20}$ These results are reasonable since the motion of terminal segments is restricted only by the linkage on one side, but the motion of the interior segments is restricted by linkages on both sides. The interior segments of the chain must restrict their motion as in the case of "crankshaftlike motion" 21,22 and others. ${ }^{23,24}$

The results shown in Figures 4 and 5 indicate that amylose in neutral aqueous solutions has more or less an ordered conformation (helix) with restricted rotation around glucoside linkage at room temperature. The ordered conformation of amylose in solution has been interpreted in terms of the "deformed helix," $17,25,26$ "interrupted helix" ${ }^{\prime, 27,28}$ or their modified models. ${ }^{29,30}$ According to the interrupted helix model, tight helical segments of the chain are essentially identical to the helix of " $V$ " amylose in the solid state. If tight helical segments having a DP 120 exist in an amylose chain as suggested by Szejtli et al. ${ }^{28}$ we might expect a large rotational relaxation time as shown from the following calculations.

Consider an amylose molecule composed of tight helical segments, each containing 120 glucose residues with a completely flexible chain between them. The flexible chain is assumed not to restrict the motion of the helical segments. If each helical segment consists of 15 helical turns, i.e., eight glucose units per turn, the rotational relaxation time for each segment would be equivalent to that for a 
long cyclindrical rod of $150 \AA$ length and $18 \AA$ diameter. A long cylindrical rod, of length $L$ and diameter $d$, can be approximated by an prolate ellipsoid of the same volume and length $(2 a=L)$ with semi-axes $a$ and $b$ satisfying the following equation, ${ }^{31}$

$$
\frac{b}{a}=\left(\frac{3}{2}\right)^{1 / 2} \frac{d}{L}
$$

The harmonic mean of the two principal relaxation times of rotation is given by,

$$
\rho_{\mathrm{h}}=\frac{3}{\left(\frac{1}{\rho_{\mathrm{a}}}+\frac{2}{\rho_{\mathrm{b}}}\right)}
$$

where $\rho_{\mathrm{a}}$ and $\rho_{\mathrm{b}}$ are the relaxation times of the axis of revolution and that perpendicular to it, respectively. $\rho_{\mathrm{a}}$ and $\rho_{\mathrm{b}}$ are calculated from, ${ }^{32}$

$$
\begin{aligned}
& \frac{\rho_{\mathrm{a}}}{\rho_{0}}=\frac{2}{3} \\
& \times \frac{1-(b / a)^{4}}{\left[2-(b / a)^{2}\right] \frac{(b / a)^{2}}{\sqrt{1-(b / a)^{2}}} \ln \frac{1+\sqrt{1-(b / a)^{2}}}{(b / a)}-(b / a)^{2}}
\end{aligned}
$$

$\frac{\rho_{\mathrm{b}}}{\rho_{0}}=\frac{4}{3}$

$$
\begin{gathered}
x \frac{1-(b / a)^{4}}{\left[1-(b / a)^{2}\right] \frac{(b / a)^{2}}{\sqrt{1-(b / a)^{2}}} \ln \frac{1+\sqrt{1-(b / a)^{2}}}{(b / a)}+1} \\
\rho_{0}=\frac{1}{k T} 4 \pi a b^{2}
\end{gathered}
$$

where $\rho_{0}$ is the rotational relaxation time of the sphere having the same volume as the ellipsoid and $k$ is the Boltzmann constant. With these equations, the $\rho_{\mathbf{h}}$ for the helical segment in water at $25^{\circ} \mathrm{C}$ is estimated to be $43 \mathrm{~ns}$. This may be the smallest value that can be expected theoretically, since completely free linkage is assumed between the tight helical segments. However, only about a quarter of this value was found experimentally (Table II). This fact suggests that amylose does not take on a tight helical conformation in an aqueous solution.

The "deformed helix" and its modified conformations proposed by several authors for amylose in

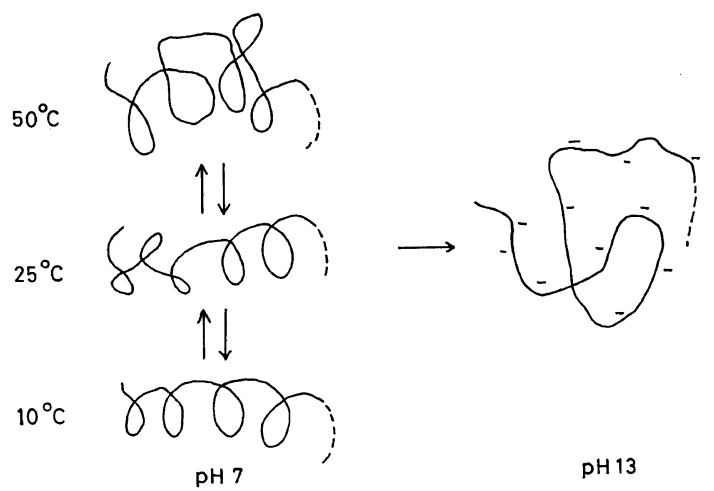

Figure 5. Model proposed for the changes in the conformation of amylose with temperature and $\mathrm{pH}$.

an aqueous solution can be approximated as those which somewhat resembling wormlike coils consisting essentially of an imperfect or deformed helical backborne. ${ }^{17,25,26,29,30}$ We consider that the increased segmental motion increases the imperfectness of the helical backborne and promotes a random-coil character of the amylose molecule throughout the chain. The "deformed helix" is thus considered to be more reasonable as a model for the conformation of amylose in an aqueous solution than "interrupted helix."

We propose a molecular mechanism illustrated schematically in Figure 5 in order to interpret the conformational change revealed by the fluorescence polarization data. The loosening of the helical structure with increasing temperature may occur first in the terminal segments at room temperature and then extend to all segments above $45^{\circ} \mathrm{C}$. In contrast to the loosening caused by thermal energy, the loosening in alkaline solution occurs in the all segments at the same $\mathrm{pH}$ due to the increased electrostatic repulsion between dissociated hydroxyl groups of glucose units.

Acknowledgements. The authors should like to thank Professor Y. Nishijima of Kyoto University for perimission to use the nanosecond fluorometer and also thank Dr. S. Ito for his help in performing the fluorescence lifetime measurements. Appreciation is due to Associate Professor M. Yamamoto of Kyoto University for his useful comments and criticism. We are indebted to Professor $\mathrm{T}$. Fukui of Osaka University for his generous gift of potato phosphorylase. 


\section{REFERENCES}

1. J. M. G. Cowie, Makromol. Chem., 42, 230 (1961).

2. W. Burchaurd, Makromol. Chem., 64, 110 (1963).

3. W. Banks and C. T. Greenwood, Makromol. Chem., 67, 49 (1963).

4. W. Banks and C. T. Greenwood, Carbohydr. Res., 7, 349 (1968).

5. W. Banks and C. T. Greenwood, "Starch and Its Components," Edinburgh University Press, Edinburgh, U.K., 1975, Chapter 4.

6. T. Kuge, "Denpun Kagaku Handbook," N. Nakamura and S. Suzuki, Ed., Asakura Shoten Co., Tokyo, 1977, pp 20-23.

7. S. Kitamura, H. Yunokawa, and T. Kuge, Polym. J., 14, 85 (1982).

8. E. V. Anufrieva, M. V. Volkenstein, M. G. Krakovyak, and T. V. Shevelava, Dokl. Akad. Nauk. $S S S R$, 186, 854 (1969).

9. T. J. Gill III and G. S. Omenn, J. Am. Chem. Soc., 87, 4188 (1965).

10. W. J. Whelan and J. M. Bailey, Biochem. J., 58, 560 (1954).

11. A. Kamogawa, T. Fukui, and Z. Nikuni, $J$. Biochem., 63, 361 (1968).

12. A. N. De Belder and K. Granath, Carbohydr. Res., 30, 375 (1973).

13. J. E. Hodge and B. T. Hofreiter, "Method in Carbohydrate Chemistry I," R. L. Whistler and M. L. Wolfrom, Ed., London, U.K., 1962, p 388.

14. G. Weber, Biochem. J., 51, 145 (1952).

15. "International Critical Tables," Vol. V, E. W.
Washburn, Ed., McGraw-Hill, 1929, p 23.

16. "Kagaku Binran," Vol. 1 (II), The Chemical Society of Japan, Ed., Maruzen Co., Tokyo, 1966, Sections $5-10$.

17. V. S. R. Rao and J. F. Foster, Biopolymers, 1, 527 (1963).

18. L. Doppert and A. J. Staverman, J. Polym. Sci., A-1, 4, 2373 (1966).

19. S. R. Erlander, R. M. Purvinas, and H. L. Griffin, Cereal Chem., 45, 140 (1968).

20. M. Uchida, M. Yamamoto, and Y. Nishijima, Polym. Prepri. Jpn., 25, 2. 297,298 (1976).

21. T. F. Schatzki, J. Polym. Sci., 57, 496 (1962).

22. E. Helfand, J. Chem. Phys., 54, 4651 (1971).

23. B. Valeur, J. P. Jarry, F. Gény, and L. Monnerie, J. Polym. Sci., Polym. Phys. Ed., 13, 667 (1975).

24. F. Gény and L. Monnerie, J. Polym. Sci., Polym. Phys. Ed., 17, 131 (1979).

25. T. Kuge and S. Ono, Bull. Chem. Soc. Jpn., 34, 1264 (1961).

26. T. Kuge, Bull. Univ. Osaka Pref., B, 11, 121 (1961).

27. J. Holló and J. Szejtli, Stärke, 10, 49 (1958).

28. J. Szejtli, M. Richter, and S. Augustat, Biopolymers, 5, 5 (1967).

29. M. Senior and E. Hamori, Biopolymers, 12, 65 (1973).

30. R. C. Jordan, D. A. Brant, and A. Cesáro, Biopolymers, 17, 2617 (1978).

31. C. Tanford, "Physical Chemistry of Macromolecules," John Wiley \& Sons, Inc., New YorkLondon, 1961, p 343.

32. F. Perrin, J. Phys. Radium, 5, 497 (1934). 